\title{
Photophysics of Przewalskinone-B and its Binding Behavior with $\beta$ - Cyclodextrin
}

Krishnaveni $\mathbf{R}^{*}$

Department of Chemistry, Saveetha School of Engineering, Saveetha University, Kuthambakkam, Tamil Nadu, India

${ }^{*}$ Corresponding author: Krishnaveni R, Department of Chemistry, Saveetha School of Engineering, Saveetha University, Kuthambakkam, Tamil Nadu, India, Tel: 0442680 1050; E-mail: krishnaveni@saveetha.com

Received: July 25, 2017; Accepted: August 12, 2017; Published: August 21, 2017

Copyright: (c) 2017 Krishnaveni R. This is an open-access article distributed under the terms of the Creative Commons Attribution License, which permits unrestricted use, distribution, and reproduction in any medium, provided the original author and source are credited.

\begin{abstract}
The photophysical properties of Przewalskinone-B, a plant extract which is reported widely for its medicinal values were studied in the absence and presence of a drug carrier, $\beta$-Cyclodextrin. The steady state absorption, fluorescence excitation and emission spectra of Przewalskinone-B were studied in an aqueous medium. The fluorescence emission of Przewalskinone-B was analysed in presence of varying concentrations of $\beta$-Cyclodextrin in an alcohol/water medium. An appreciable quenching was observed in the emission intensity of Przewalskinone-B. There was also a red shift observed in both absorption and emission spectra in presence of $\beta$-Cyclodextrin which designated a convinced interaction between the two species.
\end{abstract}

Keywords: Przewalskinone-B; $\beta$-Cyclodextrin; Emission; Binding

\section{Introduction}

For thousands of years, herbal medicine holds a great promise for medical diagnosis and treatments in Asia and now is considered a complementary or alternative medical system in most Western countries. Different from conventional medicine in which drugs are studied in isolation, herbal medicine typically incorporates several medicinal herbs which contain multiple ingredients that probably produce a more favorable response than an isolated single constituent [1].

Przewalskinone- $B$ is one such drug that is obtained from the plant Salva Perzewalskii, a herbaceous perennial one, native to the Chinese provinces of Gansu, Hubei, Sichuan, Xizang, and Yunnan, typically growing along stream banks, forest edges, among shrubs, and on granitic hillsides. It is an anthranoid form of the extract obtained from the stem bark of the genus Ochna Obtusata [2]. It is well established as a medicinal drug $[3,4]$ and is also widely acknowledged for its antiinflammatory properties [2].

The oral route of drug administration is the most convenient way of choice for the formulators and continues to dominate the area of Pharmaceutics. However, though popular, this route is limited by absorption and bioavailability in the milieu of gastrointestinal tract. Hence bioavailability is undoubtedly one of the most important pharmacokinetic parameters since it is the indicator of the efficiency of the drug delivery to the systemic circulation [1]. In spite of its availability in comfortable yields and well established medicinal properties, przewalskinone-b has got limited applications due to its poor solubility in water [5]. This directly confines its bioavailability to living species when used as a drug. Here, in our discussion we look in the direction of the enhancement of bioavailability of this drug by complexing it with a very popular drug carrier namely $\beta$-Cyclodextrin. An effective binding between the host $(\beta-C D)$ and the guest (Przewalskinone) is anticipated which would eventually increase the bioavailability of the target drug namely Przewalskinone-B.

\section{Materials and Methods}

$\beta$-Cyclodextrin used was obtained from Sigma-Aldrich and used as received. Methanol used was obtained from SD. Fine chemicals and was distilled before use. Przewalskinone-B was obtained from Pharmaceutical lab and was used as received. Water was triply distilled before usage.

Absorption spectra were recorded in an Agilent 8453 diode array spectrophotometer. Fluorescence Emission and Excitation spectra were recorded using a Horiba JobinYvonFluoromax $4 \mathrm{p}$ spectrofluorimeter [6].

\section{Results and Discussion}

\section{Photophysical analysis}

The structure of the medicinal drug [7], Przewalskinone-B is as shown in Figure 1.<smiles>COc1cc(O)c2c(c1)C(=O)c1cc(C)cc(O)c1C2=O</smiles>

Figure 1: Structure of Przewalskinone-B

The Excitation spectrum of Przewalskinone-B was recorded with 1 $\times 10^{-6} \mathrm{M}$ solution and the observation is as shown in Figure 2. Two peaks were observed at $280 \mathrm{~nm}$ and $421 \mathrm{~nm}$ which are attributed to $\mathrm{n}$ $\rightarrow \pi^{*}$ and $\pi \rightarrow \pi^{*}$ transitions happening in the molecule [8]. The 
Citation: Krishnaveni R (2017) Photophysics of Przewalskinone-B and its Binding Behavior with $\beta$-Cyclodextrin. Nat Prod Chem Res 5: 284. doi:

Page 2 of 3

absorption spectrum of Prze-B is as depicted in Figure 3 which also picturizes the two transitions at $268 \mathrm{~nm}$ and $421 \mathrm{~nm}$. The emission spectrum of the compound for the same concentration when excited at $423 \mathrm{~nm}$ showed a characteristic behavior as shown in Figure 4, which had its maximum emission intensity at $514 \mathrm{~nm}$. This shows that there occurs a single emitting species in the excited state of the Przewalskinone-B molecule.

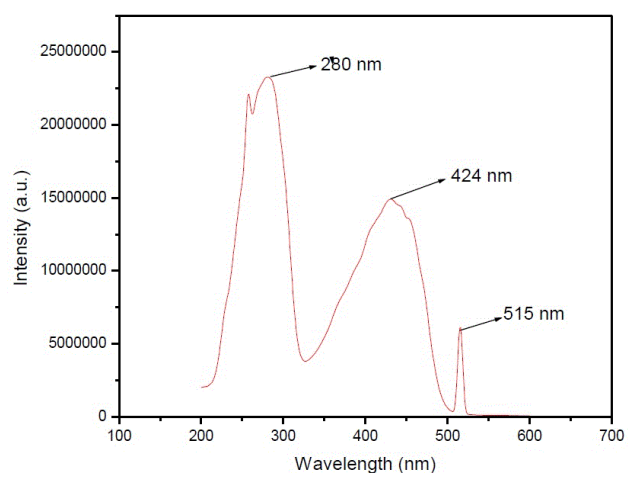

Figure 2: Excitation spectrum of Przewalskinone-B $\left[1 \times 10^{-6} \mathrm{M}\right]$ in methanol. $\lambda_{\text {emi }}=500 \mathrm{~nm}$.

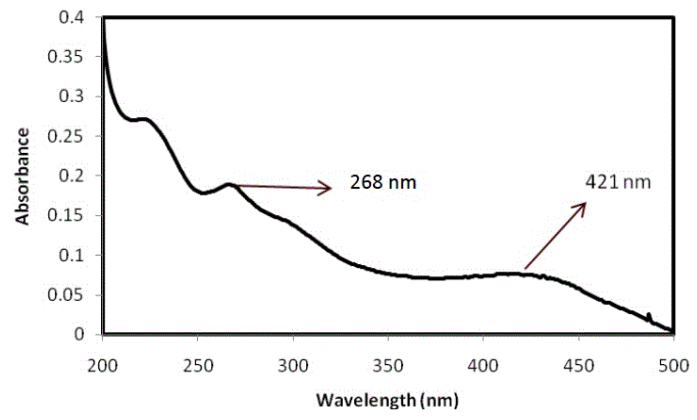

Figure 3: Absorption spectrum of Przewalskinone-B $\left[1 \times 10^{-6} \mathrm{M}\right]$ in methanol.

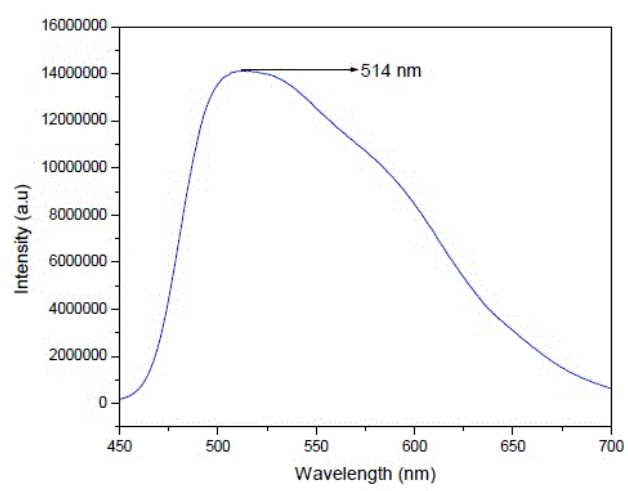

Figure 4: Steady state emission spectrum of Przewalskinone-B $[1 \times$ $10^{-6} \mathrm{M}$ ] in methanol. $\lambda_{\text {exc }}=423 \mathrm{~nm}$.

\section{Binding studies with $\beta$-cyclodextrin}

In order to understand the binding capacity of the Przewalskinone$B$ within the cavities of $\beta-C D$, binding studies were carried out by mixing a fixed concentration of Przewalskinone-B $\left(1 \times 10^{-6} \mathrm{M}\right)$ with several aliquots of $\beta$-CD ranging from $5 \times 10^{-5} \mathrm{M}, 1 \times 10^{-4} \mathrm{M}, 2 \times 10^{-4}$ $\mathrm{M}, 4 \times 10^{-4} \mathrm{M}$ and $8 \times 10^{-4} \mathrm{M}$. he corresponding absorption and emission spectra of Przewalskinone-B/ $\beta-C D$ host-guest system is as shown in Figures 5 and 6 respectively. As it is incidental from the absorption spectrum (Figure 5), there is a red shift observed with respect to absorbance maximum from $421 \mathrm{~nm}$ to $433 \mathrm{~nm}(12 \mathrm{~nm})$ which could be attributed to the inclusion of the guest molecule into the more polar hydrophobic cavity of the $\beta$-CD. Indeed, this also leads to the stability of the molecule in the excited state due to the intermolecular hydrogen bonding of the host-guest species.

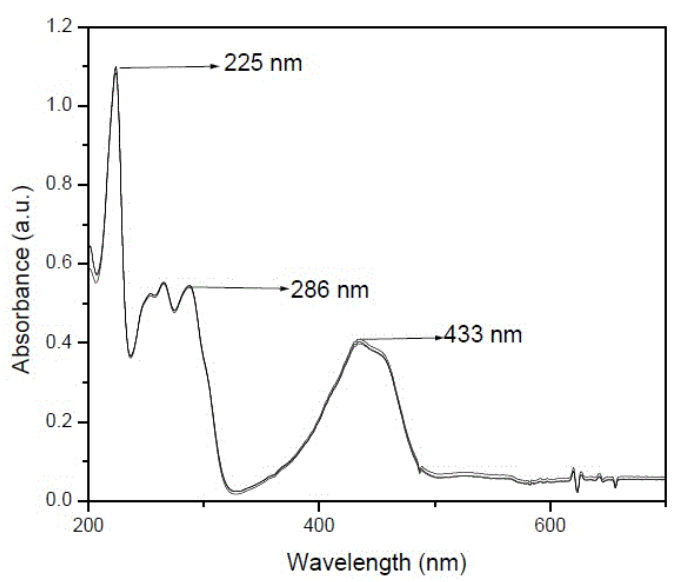

Figure 5: Absorption spectrum of Przewalskinone-B $\left[1 \times 10^{-6} \mathrm{M}\right]$ in presence of $5 \times 10^{-5} \mathrm{M}, 1 \times 10^{-4} \mathrm{M}, 2 \times 10^{-4} \mathrm{M}, 4 \times 10^{-4} \mathrm{M}$ and $8 \times$ $10^{-4} \mathrm{M}$ of $\beta$-CD.

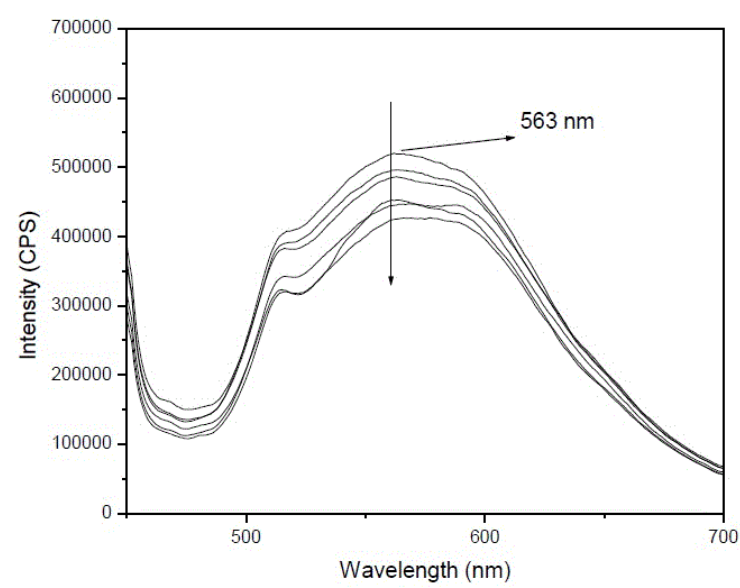

Figure 6: Emission behavior of Przewalskinone-B $\left[\begin{array}{lll}1 & \mathrm{x} & 10^{-6} \mathrm{M}\end{array}\right]$ in presence of $5 \times 10^{-5} \mathrm{M}, 1 \times 10^{-4} \mathrm{M}, 2 \times 10^{-4} \mathrm{M}, 4 \times 10^{-4} \mathrm{M}$ and $8 \times$ $10^{-4} \mathrm{M}$ of $\beta$-CD. $\lambda_{\text {exc }}=423 \mathrm{~nm}$.

The steady state emission spectrum (Figure 6) supports the above said observations as there was also a red shift observed in the spectrum 
Page 3 of 3

to the tune of around $50 \mathrm{~nm}$ and there was also a decrease in intensity of emission with increase in concentration of $\beta$-CD. Such solvatochromic shifts in inclusion complexes of cyclodextrin complexes due to the presence of a hydrophobic interior cavity have been already reported [9]. This further evidence the stability of the host-guest species due to complexion [10]. The phenomenon is pictorially represented as in Figure 7.

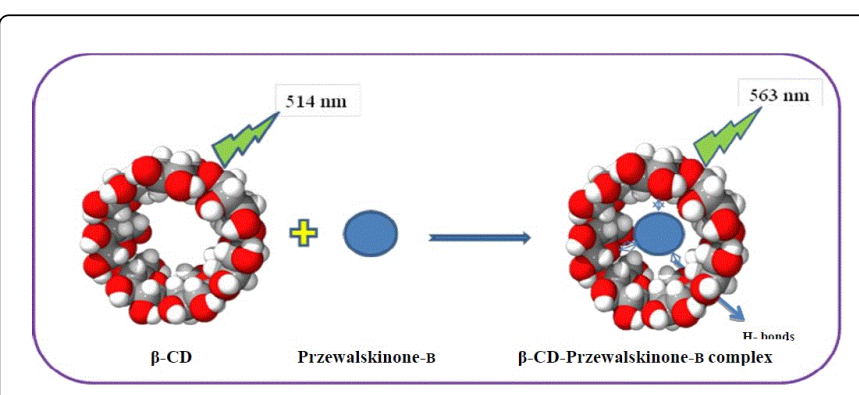

Figure 7: Effect of inclusion of Przewalskinone-B with $\beta-C D$.

\section{Determination of association constant}

Since the change in emission intensity is anticipated due to the formation of a complex between $\beta-C D$ and Przewalskinone- $B$, it is possible to use a Benesi-Hildebrand to find out the association constants of the complexes. The derivation of this method as it is applied to $\mathrm{CD}$ complexes is already reported elsewhere [11]. Accordingly, from the emission spectrum, a Benesi-Hildebrand plot was constructed (Figure 8 ) and the binding constant was determined for the host guest complex as $2 \times 10^{7} \mathrm{M}^{-1}$. The stoichiometry of complexation is assigned as a 1:1 complex from the nature of the plot as inferred from Figure 6. The high factor of association constant obtained is in good correlation with the stability of the host-guest of $\beta$ CD-Przewalskinone-B proposed [12].

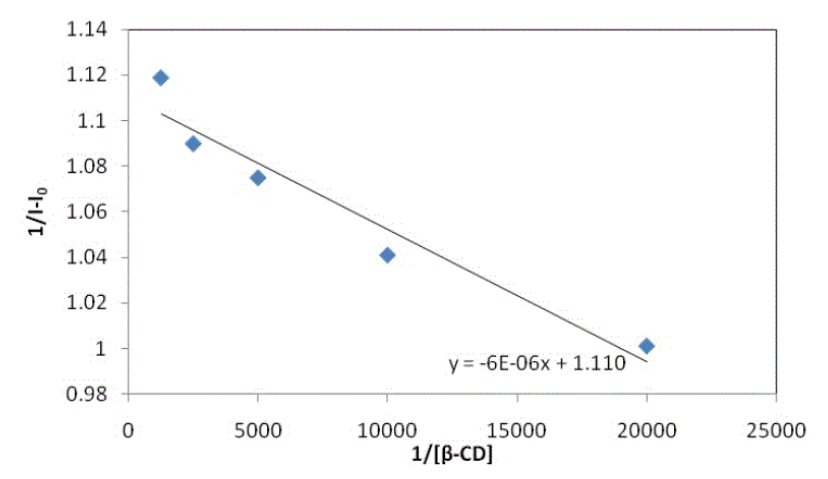

Figure 8: Benesi-Hildebrand plot of Przewalskinone-B/ $\beta-C D$ system. $\lambda_{\text {exc }}=423 \mathrm{~nm}$.

\section{Summary}

Photophysical behavior of Przewalskinone-B was recorded in an alcoholic medium. As a characteristic absorption and emission spectrum was observed, binding behavior of the molecule was analysed with a well-known host viz., $\beta$-CD. As expected, the hostguest complex was eventually formed and was also found to be highly stable when compared to the free entity of Przewalskinone- $B$ as evidenced from the red shifts observed in both absorption and emission studies of the complex. This was further supported by the high association constant obtained from Benesi-Hildebrand plot. Hence a brand-new host-guest complex of $\beta$-CD-Przewalskinone-B is hereby proposed and studied effectively.

\section{Acknowledgement}

The author would like to thank Dr. P. Ramamurthy, Director, National Centre for Ultrafast Processes for kindly providing the instrumentation facilities. Thanks, are also owed to Dr. E. Sukumar, Department of Research and Development, Saveetha University for providing the sample.

\section{References}

1. Dahanukar SA, Kulkarni RA, Rege NN (2000) Pharmacology of Medicinal Plants and Natural Products. Ind J Pharmaco 32: 81-118.

2. Sivaprakasam P, Viswanathan S, Thirugnanasambantham A (1997) Przewalskinone-B from the stem bark of Ochnaobtusata. J Indian Chem Soc 74: 165-166.

3. Xia Wang C, Xue Xu J, Weiyang Tao G, Yan Li Y, Yonghua Wang Y, et al. (2012) A Systems Biology Approach to Uncovering Pharmacological Synergy in Herbal Medicines with Applications to Cardiovascular Disease. Evidence-Based Complementary and Alternative Medicine.

4. Anil Kumar Reddy B, UngLeeb D, GhogomuTihc R, Gunasekar D, Bodoe B (2012) Phytochemical and Biological Studies of Ochna Species. Chem Biodivers 9: 251-271.

5. Schripsema J, Dagnino D (1996) Elucidation of the Substitution Pattern of Anthraquinones through the Chemical Shifts of Peri- Hydroxyl Protons. Phytochem 42: 177-184.

6. Krishnaveni R, Ramamurthy P, Padma Malar EJ, Divya P (2012) Synthesis and characterization of $\mathrm{NADH}$ model compound modi ied $\beta$ cyclodextrin and its role as an energy donor in FRET. J Photochem Photobiol A: Chem 229: 60-68.

7. Ross Kelly T, Zhenkun M, Xu W (1992) Revision of the structure of przewalskinone B. Tet Lett 33: 7713-7714.

8. Kumar S (2006) Spectroscopy of Organic Compounds. Phytochem, pp: $1-36$.

9. Nicole J, Crane Rudolph C, Mayrhofer K, homas A, Betts Gary A, et al. (2002) Cyclodextrin Inclusion Complexes with a Solvatochromic Fluorescent Probe. J Chem Ed Chem 79: 1261-1263.

10. Loftsson T, Jarho P, Masson M, Järvinen T (2005) Cyclodextrins in drug delivery. Expert Opin Drug Deliv 2: 335-351.

11. Nigam S, Durocher G (1996) Spectral and photophysical studies of inclusion complexes of some neutral $3 \mathrm{~h}$-indoles and their cations and anions with $\beta$-cyclodextrin. The J Phys Chem 100: 7135-7142.

12. Kim D, Scranton AB, Stansbury JW (2009) Analysis of Association Constant for Ground State Dye-Electron Acceptor Complex of Photo Initiator Systems and the Association Constant Effect on the Kinetics of Visible-Light-Induced Polymerizations. J PolymSci A Polym Chem 47: 1429-1439. 